\title{
Design of Minimizing Expected Energy of Multisource Wireless Cooperative Network Based on Multiobjective Optimization
}

\author{
Shusheng Wang 10 \\ Space Star Technology Co, Ltd., Beijing 100095, China \\ Correspondence should be addressed to Shusheng Wang; wangss@spacestar.com.cn
}

Received 20 January 2021; Revised 7 February 2021; Accepted 21 February 2021; Published 2 March 2021

Academic Editor: Hsu-Yang Kung

Copyright ( $\odot 2021$ Shusheng Wang. This is an open access article distributed under the Creative Commons Attribution License, which permits unrestricted use, distribution, and reproduction in any medium, provided the original work is properly cited.

\begin{abstract}
In order to solve the problems of high average power consumption, low average throughput, high average energy consumption per unit of data, and short network life cycle in traditional multisource wireless cooperation methods, this paper proposes a multisource wireless cooperative network design method based on multiple goals. We analyze the characteristics of heterogeneous deployment of multisource wireless cooperative networks and the energy consumption of nodes and control the energy consumption of network data transmission through distributed opportunistic transmission scheduling methods according to the analysis results. We use the optimal strategy of minimizing expected energy consumption, transform the problem of data transmission energy consumption, establish a mathematical model, and obtain the optimal solution for minimizing expected energy consumption. According to the optimal stop rule, the minimum expected energy consumption threshold is obtained, and then the optimal solution is obtained on the constraint set of the multiobjective optimization problem through the multiobjective optimization method, so as to achieve the goal of minimizing the expected energy of the network. Experimental results show that this method prolongs the network life cycle, reduces the average power consumption of network data transmission, and improves the average network throughput.
\end{abstract}

\section{Introduction}

As an important branch of wireless network, multisource wireless cooperative network is widely used. Especially with the use of intelligent devices, multimedia applications, and intelligent devices, the demand for network data traffic has increased sharply, and the energy consumption of the whole system has reached an unprecedented level. According to related research reports, in a typical multisource wireless cooperative network, the energy consumption caused by the operation of the base station accounts for more than $50 \%$ of the entire system [1]. Therefore, how to improve energy efficiency and reduce energy consumption is very important for multisource wireless cooperative networks. In this regard, many scholars in academia have proposed diversified solutions.

At present, the research on node energy consumption of multisource wireless cooperative network mainly focuses on topology control strategy and energy-efficient routing control strategy. The former mainly studies the reduction of the transmission energy consumption of nodes by adjusting the wireless transmission distance between nodes under the premise of ensuring the normal operation of the network [2]. The latter mainly studies the selection of suitable transmission distances and routes to store energy for sensor nodes by means of node multihop transmission. The commonality of these two types of strategies is that when the wireless transmission interface of the node is in the "active" state of transmitting/receiving information, energy consumption is reduced, but when the node is in the "idle" state, there is still energy consumption, so the sensor node sleep management strategy is put forward. Under the premise of ensuring the normal transmission of the network, this strategy shuts down some temporarily useless nodes, puts them in a "sleep" state, and only activates a few nodes for multihop transmission to save the overall energy consumption of the network $[3,4]$. Although the above strategy achieves the energy saving of network nodes, there is still the problem of large energy consumption of some nodes. 
In order to effectively reduce network latency and energy consumption, reference [5] applies Work Breakdown Structure Number (WBSN) to the Internet of Things. First of all, from the perspective of network architecture, a comprehensive study of energy saving solutions is carried out. Secondly, a mathematical model of the link and interruption probability of the protocol is established. Finally, the end-toend delay, throughput, and energy consumption are analyzed and studied. The simulation results show that, in general, compared with the direct transmission mode and existing work, this method can obtain better system performance, but the average power consumption of network data is still large after using this method; that is, the energy consumption is large. Prashanth et al. proposed a clusterbased method to minimize the energy consumption of mobile nodes in wireless sensor networks. In order to reduce the moving time of mobile nodes in wireless sensor networks, a node recognition algorithm to determine the optimal path is proposed. The data generated by the cluster members will be aggregated at the identified cluster head, and the ideal path will be determined by connecting all nodes. Experimental results show that this method can reduce node energy consumption to a certain extent, but there is a problem of short network life cycle, and it takes a lot of time to determine the optimal path. Gaujal et al. proposed a dynamic speed scaling method to minimize the expected energy consumption of real-time tasks. The discrete-time Markov decision process method is used to calculate the optimal online speed adjustment strategy to minimize the energy consumption of a single processor that performs limited or infinite multiple task sets under realtime constraints. Experimental results show that this method has better performance without considering job statistics, but the average throughput is lower, and the average energy consumption per unit data is higher.

With the rapid development of wireless network technology, saving energy consumption has become an extremely important topic in building a green wireless network. In this context, aiming at the problems existing in the existing methods, aiming at reducing the network energy consumption, improving the average throughput, and reducing the average power consumption, a multiobjective optimization based design method for multisource wireless cooperative networks is proposed.

The research contributions of this paper include the following:

(1) A multisource wireless cooperative network design method based on multiple goals is proposed.

(2) The characteristics of heterogeneous deployment of multisource wireless cooperative networks and the energy consumption of nodes are analyzed, and according to the analysis results, the energy consumption of network data transmission is controlled through the distributed opportunistic transmission scheduling method.

(3) We use the best strategy to minimize the expected energy consumption, convert the problem of data transmission energy consumption, establish a mathematical model, and obtain the best solution to minimize the expected energy consumption.

(4) According to the optimal stopping rule, the minimum expected energy consumption threshold is obtained, and then through the multiobjective optimization method, the optimal solution is obtained for the constraint set of the multiobjective optimization problem, so as to achieve the purpose of minimization.

The remaining organizational structure of the paper is arranged as follows. The second section discusses the design of minimum expected energy of multisource wireless cooperative network, the third section discusses simulation experiment, and the fourth section summarizes the whole paper.

\section{Multisource Wireless Cooperative Network Expected Energy Minimization Design}

2.1. Heterogeneous Deployment of Multisource Wireless Cooperative Network. For multisource wireless cooperative networks, energy efficiency has inevitably become a concern. Although many strategies have been continuously proposed to improve the power efficiency of the system, most of them are based on homogeneous network scenarios [6]. However, considering the increasing user rate requirements, the structure of the multisource wireless cooperation network has gradually shifted to a heterogeneous network architecture. Figure 1 is an example diagram of the heterogeneous network deployment of the multisource wireless cooperation network.

According to the different network scenarios, the optimization of energy consumption in multisource wireless cooperative networks can be divided into two aspects: the unknown network topology and the known network topology. In the former, base stations are effectively deployed within the specified region to achieve network coverage and reduce energy consumption [7]. If the microbase station is deployed in the macrocell with high density without plan, although it can provide high data rate, it will inevitably lead to a sharp increase in energy consumption.

On this basis, under the condition that the whole network structure is fixed, the performance of the multisource wireless cooperative network and the efficiency of the base station are improved by rational allocation of network resources, so as to achieve the purpose of energy saving.

\subsection{Description of Node Energy Consumption Problem.} According to the heterogeneous network layout of multisource wireless cooperative network, the energy consumption of multisource wireless cooperative network nodes has timevarying characteristics. In order to meet the receiving power requirements of the receiving terminal, it is necessary to send data with a higher power, which will lead to the decline of energy utilization. A reasonable way to solve this problem is to let some nodes give up this transmission opportunity, and let all nodes compete again, so that the link that successfully obtains 


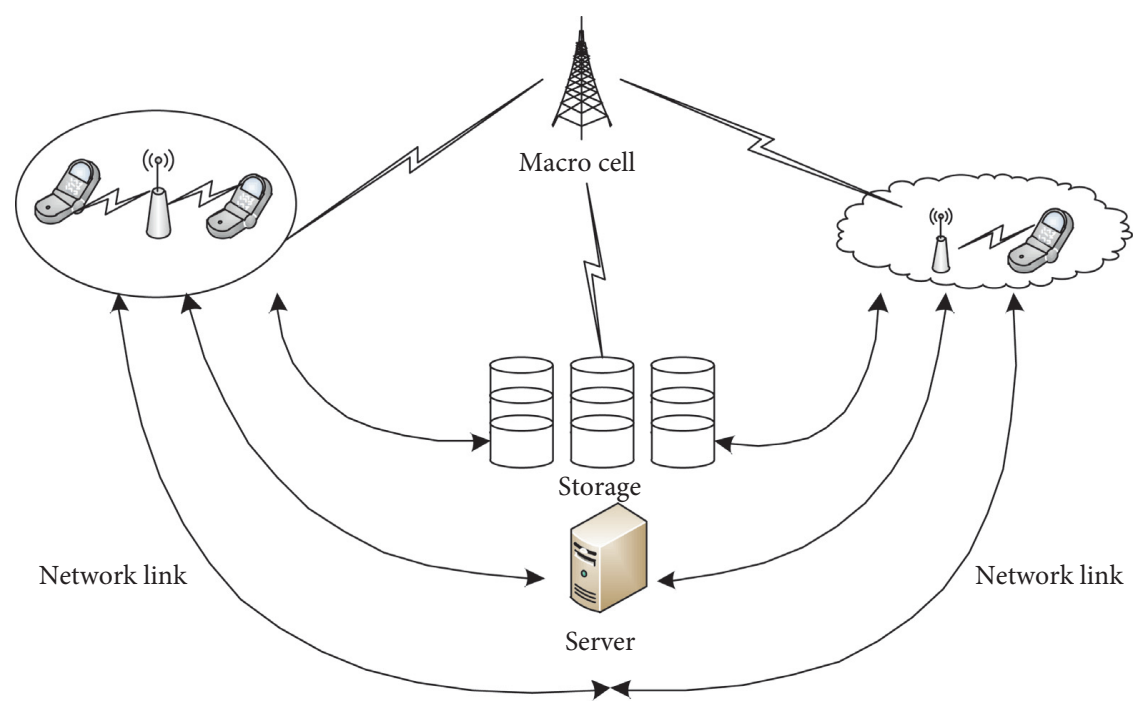

FIGURE 1: Example diagram of heterogeneous layout of multi-source wireless cooperative network.

the channel after recompetition can transmit data in a better channel state [8]. Because different links experience different channel state changes at different times, after further channel detection, it is possible for links to obtain better channel state. Therefore, the diversity of users and the diversity of channel fluctuation can be used to optimize the network performance [9]. On the other hand, each additional channel detection will cause data delay, which is originally used to transmit data. Considering this problem, an optimal stop rule is needed to detect the channel to minimize the network energy consumption.

Assuming that the maximum number of acceptable channel detections in one transmission is $m$, the upper limit of the time that the sending terminal can delay data transmission is $T_{\max }$. The calculation formula is

$$
T_{\max }=k_{1} \mu+k_{2} \mu+\cdots+k_{m} \mu .
$$

in which $k$ represents the actual delay; $\mu$ represents the external clock. The problem studied in this paper is to determine when it is best to start transmitting data within the time range $\left[0, T_{\max }\right]$.

Therefore, according to the optimal stopping theory, the duration of energy minimization is obtained, and the stop moment of energy minimization is set to $t$, and then the duration of energy minimization is

$$
t_{s}=\sum_{m=1}^{n} k_{i} \mu .
$$

Among them, $n$ represents the number of links. This duration is the time that the scheduling strategy delays this communication, which is defined as the scheduling delay. If the delayed time arrives at $T_{\max }$, and the data has not been sent yet, at this time, regardless of the channel status, the multisource wireless cooperative network will send data at time $T_{\max }$.

To sum up, in the process of two-continuous-channel detection, the communication module of the sending terminal can be set to idle mode or sleep mode to avoid its continuous work. In this way, the energy consumption can be reduced.

\subsection{Distributed Opportunistic Transmission Scheduling.} The main goal of this paper is to minimize the energy consumption of data transmission in a multisource wireless cooperative network. In order to achieve this goal, we use the good timing of the channel to construct a distributed opportunistic transmission scheduling strategy [10]. In a multiuser multisource wireless cooperative network, there are $n$ links, and mutual communication between nodes will cause interference. The nodes access the channel through the carrier sense multiple access/conflict avoidance mechanism. Figure 2 shows the minimization of multiple channels and source wireless collaborative network system model.

Since the analysis in this paper is mainly focused on the energy-efficient communication problem of the wireless link in the entire multisource wireless cooperative network, the system model only considers the impact of small-scale fading on the communication link and assumes that the communication channel will compete successfully for the link. The sending terminal must send data within the specified time range, the delay time of the sending terminal sending data cannot exceed the specified upper limit of time, and the transmission rate is constant; that is, the receiving power of the receiving terminal is fixed [11]. It is stipulated that there is always data that can be transmitted in the communication environment; that is, when the terminal device decides to transmit the data, there is always data that can be obtained. These data can be stored in the device's memory or the device can get it instantly.

\subsection{Optimal Strategies for Minimizing Expected Energy} Consumption. Using $E$ to represent the energy consumption of data transmission in a multisource wireless cooperative network, the optimization problem can be expressed as an optimal stopping problem of selecting a stopping rule 


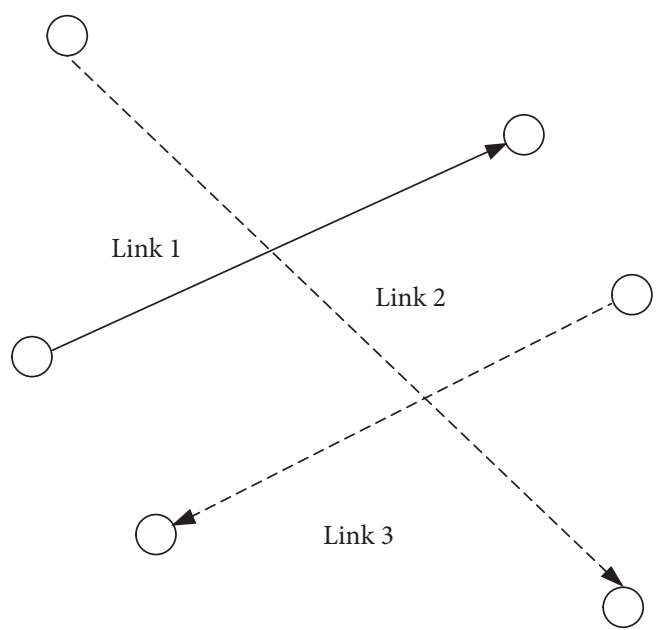

FIGURE 2: Multisource wireless cooperative network system model.

$1 \leq N \leq m$ to minimize the expected energy consumption $E_{j}$ of the network. In order to make the multisource wireless cooperative network system model more in line with the actual situation, it is assumed that the sending terminal consumes a certain amount of energy during each channel competition, which is defined as $E_{l}$. Therefore, the total data transmission energy consumption $E$ in the network is the sum of the total energy consumed by channel detection and the power consumption $P_{t}$ of transmitting data at time $t$. Then, $E$ can be expressed as

$$
E=\sum_{m=1}^{n}\left[t_{s} \times\left(E_{l}-E_{j}\right)\right]^{2}-u_{e} u_{t} \times G_{n} .
$$

Among them, $u_{t}$ represents the channel detection time; $G_{n}$ represents the channel gain sequence, which represents a random variable; $u_{e}$ represents the energy consumed by the channel detection, and its value is 1 or 0 . When it is 0 , the link does not participate in channel competition. When it is 1 , it means that the link participates in channel competition.

According to formula (3), the optimal stopping rule can be derived from the channel gain sequence and data transmission energy consumption.

The problem of data transmission energy consumption in the multisource wireless cooperative network has been transformed into the problem of minimizing the expected energy consumption, and the corresponding mathematical model has been established [12]. Before solving this problem, it is first necessary to prove that there is an optimal solution to the problem of minimizing expected energy consumption. Therefore, the following propositions are given.

Proposition 1. Formula (3) has an optimal stopping rule.

Proof. The optimal stopping rule exists when the following two conditions are met:

$$
\left\{\begin{array}{l}
S_{1}: E\left[\inf _{n} E\right]>-\infty \\
S_{2}: \lim \inf E \geq E_{\infty}
\end{array}\right.
$$

Consider these two conditions separately below:
The random variable $G_{n}$ is independently distributed, and its domains are all finite values. Therefore, $E>-\infty$ is established; that is, the $E\left[\inf _{n} E\right]>-\infty$ condition is satisfied.

When $n \longrightarrow \infty, E \longrightarrow \infty$, and the random variable $G_{n}$ is independently distributed, and the domains are all finite values, then

$$
\lim \inf E=\lim \inf \left(\frac{S}{G_{n}} t+N E\right)=\infty .
$$

According to formula (5), it can be known that $E_{\infty}=\infty$, and then $\liminf E \geq E_{\infty}$ condition is satisfied. This proves Proposition 1.

2.5. Obtaining the Minimum Expected Energy Consumption Threshold Based on the Optimal Stopping Rule. Comprehensive analysis of node energy consumption and expected energy consumption minimization optimization strategy shows that after the link obtains the channel state at any time, it must decide whether this time is the best stop time for data transmission, and whether to stop or continue detection depends on whether there is a better channel state expectation in the future [13]. The problem of limited scope can usually be solved by backward induction from the final stage to the initial stage. The optimal strategy of expected energy consumption minimization mentioned in the previous section can be deduced by comparing the sending terminal with the threshold sequence in each stage $t_{k}$ and then deciding whether to transmit data or continue detection. The minimum energy consumption cost of detection channel $x$ at a specific time $t_{k}$ is given as follows:

$$
E_{\min }=\min \left(P_{t j}, W_{i-j}\right)+E_{i j}
$$

Among them, $W_{i-j}$ represents the expected cost that can be obtained by continuing channel detection. It can be seen that formula (6) compares the expected cost from time $i$ to time $j$ with the cost $P_{t j}$ obtained by stopping at time $t$ and continuing to use the optimal stopping rule and considers the detection energy consumption cost $E$ required by continuing to detect at each stage; $W_{i-j}$ depends only on the number of moments $i-j$ to continue detection. Therefore, the optimal stopping rule is as follows: at time $j$, if $P_{t j} \leq W_{i-j}$, the sending terminal stops detecting and transmitting data; otherwise, it gives up this channel transmission opportunity and continues channel detection.

The optimal stopping rule means that, at each moment, $j$ has a transmission power threshold, $j=\left(W_{i-j}\right) / T$. The "optimal" mentioned in this article means that if the multisource wireless cooperative network stops detection after a certain round of channel detection, then this indicates that it believes that it can obtain it even if it continues to detect the channel until the maximum number of detections. The cost of energy consumption in multisource wireless cooperative network is no less than the cost of data acquisition from base station. Therefore, for multisource wireless cooperative network, the station can obtain the minimum energy consumption in the whole channel detection process, that is, the optimal energy consumption [14]. 
The real-time energy consumption cost of multisource wireless cooperative network at a certain time is an objective reflection of the channel state and detection times obtained by channel detection, while the expected energy consumption cost obtained by the next channel detection is calculated dynamically according to the current channel state and detection times [15].

Setting $P_{\theta}$ represents the maximum output transmission power of the RF power amplifier of the transmitting terminal, and $A_{\tau}$ represents the cumulative distribution function of transmission power relative to $P_{\theta}$. In order to ensure that the maximum transmission delay is $T_{\text {delay }}$, when $j=m$, set $A_{0}=P_{\theta} \times T_{i}$. Then, the $W_{i-j}$ at each stage can be obtained by reverse induction:

$$
W_{i-j}=S(m, n) W(i+\tau, j+\tau) \mathrm{d} t e^{-j \omega \tau} \mathrm{d} \tau .
$$

Among them, $S$ represents the network survival time; $\omega$ represents the number of cooperative nodes; $d$ represents the network transmission load; $\tau$ represents the density of relay nodes.

According to the proposed model, combined with the requirements of the optimal stopping rule, the threshold for minimizing the expected energy consumption at each moment is obtained:

$$
P_{r t}=\frac{E(P(u, v))}{W_{i-j}} .
$$

Among them, $u$ represents the average energy consumption of data transmission; $v$ represents the data transmission rate. According to the obtained energy threshold, in order to further realize the expected energy minimization of multisource wireless cooperative network, according to the threshold, the multiobjective optimization method is used to obtain the optimal energy minimization threshold.

\subsection{Realization of Network Expected Energy Minimization} Based on Multiobjective Optimization. At present, the commonly used optimization methods include analytical method, numerical method, hybrid method, and intelligent optimization algorithm. For the objective function and constraints in the optimization mathematical model, if there is a clear mathematical analysis expression, the final result can be obtained by derivative method or variational method according to the necessary conditions of function extremum. Analyze the solution, and then determine the optimal solution of the problem according to the actual physical meaning of the problem. This is the simplest analytical method [16].

If the objective function or constraint conditions are more complicated, or there is no clear mathematical analysis expression, or the existing analytical methods and means cannot be used to obtain the optimization problem of the analytical solution, it can be solved by the numerical solution method.

Currently, with the rapid development of computers in this era, numerical algorithms have shown their superiority. The basic idea is to use a search method to go through a series of iterations, so that the generated sequences can gradually approach the optimal solution of the problem.
Numerical solutions often require experience or experimentation. At the same time, the results also need to be verified by actual problems to be effective [17]. The hybrid solution is a combination of the above two methods, such as a type of solution represented by the gradient method, which is often a combination of analytical and numerical algorithms. If the optimization problem has particularly complex constraints and objective functions, the above solution method can no longer obtain the optimal solution. At this time, a multiobjective optimization method is needed to obtain the optimal solution.

This paper uses a multiobjective optimization method to convert the single objective in the network expected energy minimization problem to multiple objectives and uses a linear accumulation function as the evaluation function to solve the multiobjective optimal result [18].

The linear weighting method is to multiply a set of weight coefficients according to the importance of $M$ objectives and add them as the objective function, and then the objective function is to find the optimal solution on the set of constraints of the multiobjective optimization problem, that is, to construct the following multiobjective optimization problem:

$$
E_{\min }=\sum_{i=1}^{l} \xi_{i}\left(w \cdot \varphi\left(x_{i}\right)\right)+b
$$

Among them, $\zeta_{i}$ represents the total energy consumption of the network; $\varphi$ represents the network connectivity; $x_{i}$ represents the node energy consumption balance; $b$ represents the multiobjective optimization function.

By solving the optimal solution of the multiobjective optimization problem, the optimal solution of the multiobjective optimization problem in the sense of linear weighting is obtained:

$$
G_{s c}=G_{0}+\frac{E_{\min }}{\left(c_{s}-a P_{n}\right)\left(1+\left(F / F_{0}\right)\right)} .
$$

Among them, $G_{0}$ represents the linear weighted norm; $P_{n}$ represents the utility function of the node's transmission power; $F_{0}$ represents the remaining energy of the node; $a$ represents the node's movement overhead when the network transmits power; $c_{s}$ represents the weight coefficient. The weight coefficient in formula (10) satisfies the following conditions:

$$
c_{s}= \begin{cases}1, & \left|P_{k}(x, y)-P_{0}(x, y)\right|, \\ 0, & \text { others. }\end{cases}
$$

Among them, $P_{k}(x, y)$ represents the weighting coefficient of the network energy function; $P_{0}(x, y)$ represents the weighting coefficient of the energy distribution. Under the condition that formula (11) is satisfied, the expected energy minimization result of the multisource wireless cooperative network is obtained.

\section{Simulation Experiment}

In order to verify the feasibility and effectiveness of the multisource wireless cooperative network design method 
based on multiobjective optimization, the following simulation experiments are designed.

In order to avoid the singleness of the experimental results and enhance their contrast, this study introduces the idea of comparative experiment and obtains the comparison results between the method and the traditional method. On this basis, the effectiveness of the simulation results is analyzed (Table 1).

\subsection{Experimental Environment and Scheme}

(1) Experimental hardware environment setting and parameter setting: the simulation experiment is carried out on a computer with Windows 10 operating system, quad-core $3.75 \mathrm{GHz} \mathrm{CPU}, 16 \mathrm{~GB}$ RAM, and MATLAB 2014a is used to process the experimental data. The specific experimental parameter settings are shown in Table 1.

(2) Experimental comparison methods: select the network energy minimization method based on WBSN (reference [5] method), cluster-based wireless sensor network mobile node travel time minimizing energy consumption method (reference [19] method), and a method of minimizing expected energy consumption based on dynamic speed scaling to minimize real-time tasks (reference [20] method) as comparison methods.

(3) Experimental index setting

The average power consumption, network lifetime, average energy consumption per unit data, and average throughput of network data transmission are taken as experimental indexes to verify the application effect of different methods. Among them, the most important evaluation indexes are average power consumption and average throughput. Another key index is the average energy consumption per unit data, which is used to measure whether the system is efficient and energy-saving during data transmission. The lower the index, the higher the energy efficiency of the system, and the lower the energy consumption. The unit of average energy consumption per unit of data is joules per megabyte, that is, watts per megabyte per second. The calculation formula is as follows:

$$
P_{\text {cost }}=\frac{\mathrm{PC}}{\mathrm{THR}} \text {. }
$$

The calculation formula of average power consumption is as follows:

$$
\mathrm{PC}=\frac{P_{n} t+E}{\sum_{i=1}^{l} \alpha_{i} y_{i}-P_{k}(x, y)} .
$$

Among them, $\alpha_{i}$ represents the optimal allocation result of active power; $y_{i}$ represents the energy consumption of single data transmission.

The average throughput is calculated as follows:

$$
\mathrm{THR}=\sum_{j=1}^{l} \alpha_{j} y_{j}(a+b) .
$$

Among them, $\alpha_{j}$ represents the forward link data throughput; $y_{j}$ represents the reverse link data throughput.

According to the calculation formula given, calculate the numerical value of each experimental index and compare the application effects of different methods.

\subsection{Analysis of Experimental Results}

3.2.1. Comparison of Network Life Cycle. Taking the network life cycle as the experimental index, the different methods are compared, and the results are shown in Table 2.

Analysis of the data in Table 2 shows that when the number of iterations is 50, the network life cycle of the proposed method is 16.10, the network life cycle of the method in reference [5] is 7.25, the network life cycle of the method in reference [19] is 7.78, and the network life cycle of the method in reference [20] is 2.82; when the number of iterations is 100, the network life cycle of the proposed method is 20.99 , the network life cycle of the method in reference [5] is 10.88 , the network life cycle of the method in reference [19] is 9.28, and the lifetime of the network of the method in reference [20] is 4.31. According to the above data analysis results, the network life cycle of the proposed method is significantly higher than that of the traditional method, indicating that the method can improve the life cycle of a multisource wireless cooperative network [21].

3.2.2. Comparison of Average Power Consumption. Take average power consumption as the experimental index, compare different methods, and the result is shown in Figure 3.

Analysis of Figure 3 shows that when the number of iterations is from 0 to 3 , the average power consumption of the proposed method is not superior, even higher than that of the traditional method, but since the third iteration, the proposed method has always maintained its superiority [22]. The average power consumption is less than $3000 \mathrm{~W}$, and reference [5] method has a higher average power consumption when the number of iterations is less than 12; although it is reduced later, it is still higher than the proposed method [23]. The method of reference [19] and the method of reference [20] have similar trends but still have a certain gap with the proposed method. This is because the method reduces the average power consumption of the multisource wireless cooperative network through the optimization strategy of expected energy consumption minimization, thereby improving the application effect of the method [24].

3.2.3. Comparison of Average Throughput. Taking the average throughput as the experimental index, the different methods are compared, and the results are shown in Figure 4. 
TABLE 1: Experimental parameter settings.

\begin{tabular}{lc}
\hline Parameter & Value \\
\hline Number of links & Article 75 \\
Link capacity & $1500 \sim 2000$ \\
Number of link requests generated & Article $100 \sim 200$ \\
Link resources between the core layer and the aggregation layer & 550 \\
Node reading & 3 \\
Service request time slot length & $5 \mathrm{~min}$ \\
The number of nodes supporting the same function type & 8 \\
Number of web services generated & 500 \\
Node capacity & $1000 \sim 1500$ \\
\hline
\end{tabular}

TABLe 2: Comparison of network life cycles under different methods.

\begin{tabular}{lcccc}
\hline \multirow{2}{*}{ Iterations (time) } & \multicolumn{3}{c}{ Network life cycle } \\
& The proposed method & The method in [5] & The method in [19] & The method in [20] \\
\hline 10 & 15.52 & 4.31 & 6.47 & 1.58 \\
20 & 14.88 & 5.12 & 7.77 & 7.09 \\
30 & 16.96 & 6.09 & 7.15 & 2.45 \\
40 & 16.37 & 6.13 & 7.78 & 2.74 \\
50 & 16.10 & 7.25 & 8.13 & 3.74 \\
60 & 17.93 & 8.62 & 9.14 & 3.19 \\
70 & 18.52 & 8.96 & 9.19 & 3.95 \\
80 & 18.58 & 9.97 & 9.28 & 4.05 \\
90 & 19.69 & 10.78 & 10.88 & 4.31 \\
\hline 100 & 20.99 & &
\end{tabular}

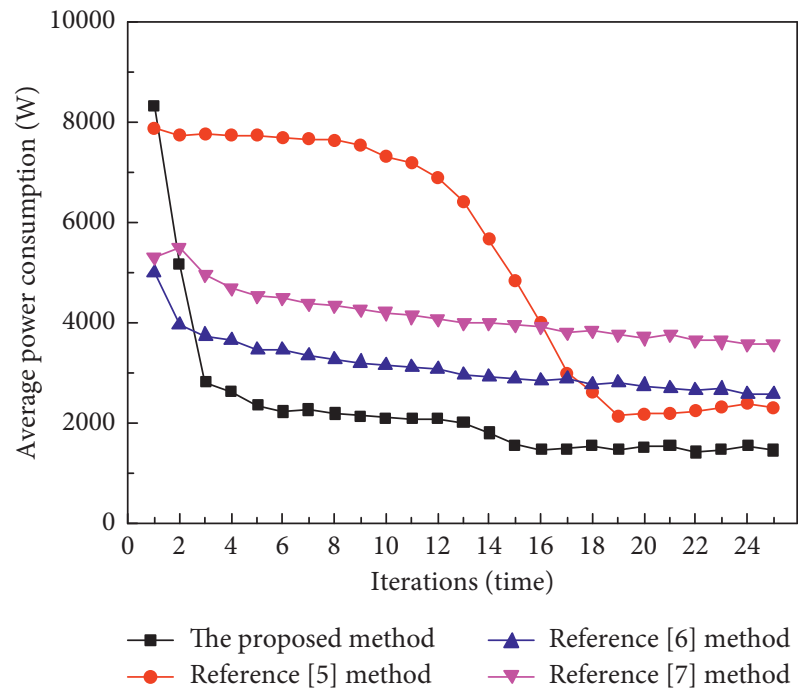

Figure 3: Comparison of average power consumption of different methods.

Analysis of Figure 4 shows that the average throughput of the proposed method is always higher than that of the traditional method, and its highest average throughput is close to $300 \mathrm{bit} / \mathrm{s}$, while the highest average throughput of the traditional method is not higher than $250 \mathrm{bit} / \mathrm{s}$. The average throughput is at a low level when the number of iterations is less than 8 times. Through comparison, it can be seen that the proposed method has significant advantages in terms of average throughput [25].

This is because the method uses the distributed opportunistic transmission scheduling method to measure the

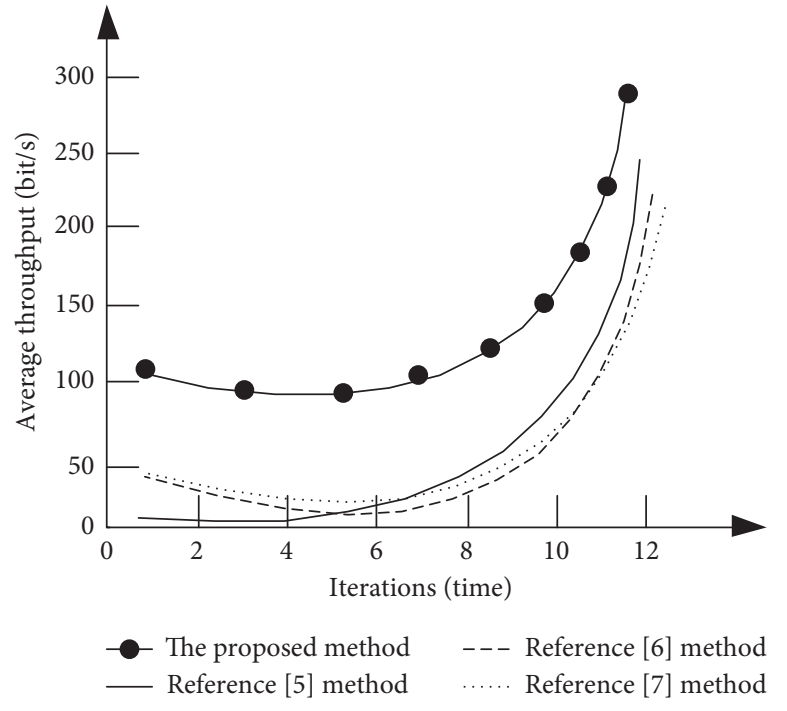

FIGURE 4: Comparison of average throughput of different methods.

energy consumption of data transmission in the multisource wireless cooperative network according to the results of the node energy consumption problem description. Research has improved the average throughput of a multisource wireless cooperative network while ensuring that the receiving terminal's received power is fixed.

3.2.4. Comparison of Average Energy Consumption per Unit Data. Taking the average throughput as the experimental index, the different methods are compared, and the results are shown in Figure 5. 


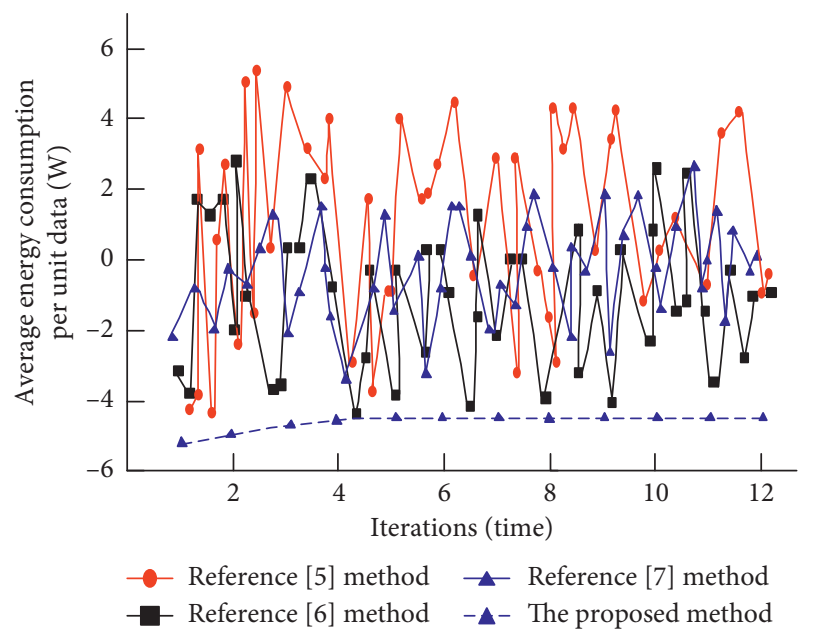

Figure 5: Comparison of average energy consumption per unit data of different methods.

Analysis of the results shown in Figure 5 shows that the average energy consumption per unit data of this method is significantly lower than that of the three traditional methods, and this advantage will not weaken with the increase of the number of iterations. However, the average unit energy consumption data of traditional methods show extremely irregular changes. Therefore, the average unit energy consumption data of this method can maintain a relatively stable level, which proves the effectiveness of this method.

The results show that the energy consumption of data processing in multisource wireless cooperative network is low under the proposed method; that is, the method effectively reduces the expected energy of multisource wireless cooperative network and achieves the research purpose of minimizing the expected energy.

In summary, the experimental results show that the proposed method has obvious advantages in average energy consumption per unit data and average throughput. It can optimize the energy of multisource wireless cooperative network, reduce the energy consumption, and realize the network optimization.

\section{Conclusion}

With the rapid development of wireless networks and data services, how to effectively manage network resources becomes more and more important. In many cases, the battery capacity of wireless devices is limited, especially in the noninfrastructure network composed of wireless devices, there is no continuous power supply, and the mobility of wireless devices reduces the opportunity of power access.

Therefore, it is of great significance to reduce the energy consumption of wireless network, and it is also an important topic to build a green network. Under this background, a design method of multisource wireless cooperative network based on multiobjective optimization is proposed, and the main results of this method are as follows:

(1) According to the characteristics of heterogeneous distribution of multisource wireless cooperative network, the energy consumption of nodes is described. According to the analysis results, the distributed opportunistic transmission scheduling method is used to study the energy consumption of network data transmission. This method can improve the average throughput of multisource wireless cooperative network.

(2) Using the optimization strategy of expected energy consumption minimization, the problem of data transmission energy consumption is transformed into the problem of expected energy consumption minimization, and the mathematical model is established to obtain the optimal solution of expected energy consumption minimization.

(3) The minimum expected energy consumption threshold is obtained by the optimal stop rule, and then the optimal solution is obtained on the constraint set of the multiobjective optimization problem by the multiobjective optimization method to complete the network expected energy minimization design.

(4) Experimental results show that the proposed method achieves satisfactory energy-saving effect and significantly improves the network energy efficiency, which is of great significance to the research of wireless network energy efficiency.

Although the design method of this study has achieved some results, due to the limitation of research time, there are some shortcomings in the research. Therefore, in the next research, the design method of this study will be further optimized to further reduce the energy consumption of wireless networks with MIMO links.

\section{Data Availability}

The data used to support the findings of this study are included within the article.

\section{Conflicts of Interest}

The authors declare that they have no conflicts of interest.

\section{References}

[1] L. C. Mutalemwa, S. Shin, and E. Sciubba, "Secure routing protocols for source node privacy protection in multi-hop communication wireless networks," Energies, vol. 13, no. 2, p. 292, 2020.

[2] M. Mousavi, H. Alshatri, and A. Klein, "Cost sharing games for energy-efficient multi-hop broadcast in wireless networks," IEEE Transactions on Wireless Communications, vol. 19, no. 1, pp. 310-324, 2019.

[3] L. T. T. Hoc, H.-S. Nguyen, Q.-P. Ma et al., "Outage and bit error probability analysis in energy harvesting wireless cooperative networks," Elektronika ir Elektrotechnika, vol. 25, no. 5, pp. 69-74, 2019.

[4] A. Bhowmick, G. C. Das, S. D. Roy, S. Kundu, and S. P. Maity, "Allocation of optimal energy in an energy-harvesting 
cooperative multi-band cognitive radio network," Wireless Networks, vol. 26, no. 2, pp. 1033-1043, 2020.

[5] A. Alkhayyat, A. A. Thabit, F. A. Al-Mayali, and Q. H. Abbasi, "WBSN in IoT health-based application: toward delay and energy consumption minimization," Journal of Sensors, vol. 2019, no. 5, pp. 1-14, 2019.

[6] J. C. Kwan and A. O. Fapojuwo, "Performance optimization of a multi-source, multi-sensor beamforming wireless powered communication network with backscatter," IEEE Sensors Journal, vol. 19, no. 22, pp. 10898-10909, 2019.

[7] J. Qadir, A. Khan, M. Zareei, and C. Vargas-Rosales, "Energy balanced localization-free cooperative noise-aware routing protocols for underwater wireless sensor networks," Energies, vol. 12, no. 22, p. 4263, 2019.

[8] R. M. Rizk-Allah, A. E. Hassanien, and A. Slowik, "Multiobjective orthogonal opposition-based crow search algorithm for large-scale multi-objective optimization," Neural Computing and Applications, vol. 32, no. 17, pp. 13715-13746, 2020.

[9] A. Rostamian, S. Jamshidi, and E. Zirbes, "The development of a novel multi-objective optimization framework for nonvertical well placement based on a modified non-dominated sorting genetic algorithm-II," Computational Geosciences, vol. 23, no. 5, pp. 1065-1085, 2019.

[10] K. Kaur, S. Garg, G. Kaddoum, E. Bou-Harb, and K.-K. R. Choo, "A big data-enabled consolidated framework for energy efficient software defined data centers in IoT setups," IEEE Transactions on Industrial Informatics, vol. 16, no. 4, pp. 2687-2697, 2020.

[11] D. Nguyen Quoc, L. Bi, Y. Wu, S. He, L. Li, and D. Guo, "Energy efficiency clustering based on Gaussian network for wireless sensor network," IET Communications, vol. 13, no. 6, pp. 741-747, 2019.

[12] L. M. Cruz, D. L. Alvarez, A. S. Al-Sumaiti, and S. Rivera, "Load curtailment optimization using the PSO algorithm for enhancing the reliability of distribution networks," Energies, vol. 13, no. 12, pp. 3236-3251, 2020.

[13] A. Ouali, D. Allouche, S. De Givry et al., "Variable neighborhood search for graphical model energy minimization," Artificial Intelligence, vol. 278, no. 1, pp. 103194.1-103194.22, 2020.

[14] P. Hoyingcharoen and W. Teerapabkajorndet, "Expected probabilistic detection and sink connectivity in wireless sensor networks," IEEE Sensors Journal, vol. 19, no. 12, pp. 4480-4493, 2019.

[15] R. Arthi, V. Jagapathi Babu, T. Aditya, and Y. Akshay Kumar, "Queue stability and low energy for energy harvesting cognitive radio networks," The International Journal of Electrical Engineering \& Education, vol. 56, no. 4, pp. 338-347, 2019.

[16] M. Liaqat, K. A. Noordin, T. Abdul Latef, and K. Dimyati, "Power-domain non orthogonal multiple access (PD-NOMA) in cooperative networks: an overview," Wireless Networks, vol. 26, no. 1, pp. 181-203, 2020.

[17] C. Wang, "Simulation of energy hole attack suppression in non-uniform node distributed networks," Computer Simulation, vol. 37, no. 4, pp. 322-326, 2020.

[18] N. Javaid, U. Shakeel, A. Ahmad, N. Alrajeh, Z. A. Khan, and N. Guizani, "DRADS: depth and reliability aware delay sensitive cooperative routing for underwater wireless sensor networks," Wireless Networks, vol. 25, no. 2, pp. 777-789, 2019.

[19] J. S. Prashanth and S. V. Nandury, "A cluster-based approach for minimizing energy consumption by reducing travel time of mobile element in WSN," International Journal of
Computers Communications \& Control, vol. 14, no. 6, pp. 691-709, 2019.

[20] B. Gaujal, A. Girault, and S. Plassart, "Dynamic speed scaling minimizing expected energy consumption for real-time tasks," Journal of Scheduling, vol. 23, no. 10, pp. 1-20, 2020.

[21] F. Wen, Y. Zhao, M. Zhang, and C. Hu, "Forecasting realized volatility of crude oil futures with equity market uncertainty," Applied Economics, vol. 51, no. 59, pp. 6411-6427, 2019.

[22] J. Cao and F. Wen, "The impact of the cross-shareholding network on extreme price movements: evidence from China," Journal of Risk, vol. 22, no. 2, pp. 79-102, 2019.

[23] D. Ciuonzo and P. Salvo Rossi, "Distributed detection of a non-cooperative target via generalized locally-optimum approaches," Information Fusion, vol. 36, pp. 261-274, 2017.

[24] W.-T. Sung and M.-H. Tsai, "Multi-sensor wireless signal aggregation for environmental monitoring system via multibit data fusion," Applied Mathematics \& Information Sciences, vol. 5, no. 3, pp. 589-603, 2011.

[25] X. Cheng, D. Ciuonzo, and P. S. Rossi, "Multibit decentralized detection through fusing smart and dumb sensors based on Rao test," IEEE Transactions on Aerospace and Electronic Systems, vol. 56, no. 2, pp. 1391-1405, 2019. 